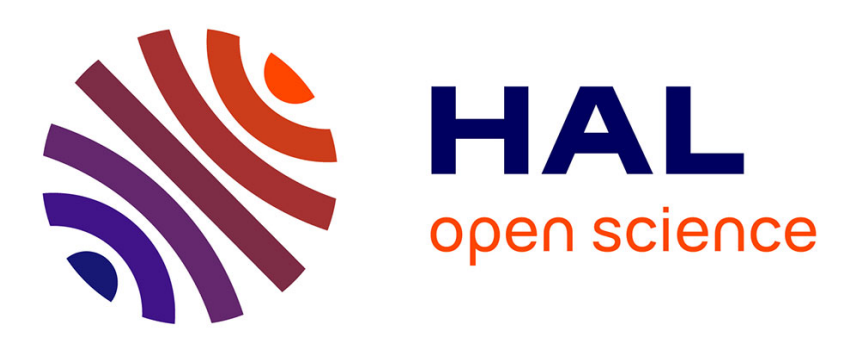

\title{
Time-frequency analysis to detect bone fracture in impact biomechanics. Application to the thorax
}

François Gabrielli, Damien Subit, Erick Ogam, Philippe Guillemain, Richard

Kent, Catherine Masson

\section{- To cite this version:}

François Gabrielli, Damien Subit, Erick Ogam, Philippe Guillemain, Richard Kent, et al.. Timefrequency analysis to detect bone fracture in impact biomechanics. Application to the thorax. Medical Engineering \& Physics, 2009, 31 (8), pp.952-958. 10.1016/j.medengphy.2009.05.007 . hal-00849434

\section{HAL Id: hal-00849434 \\ https://hal.science/hal-00849434}

Submitted on 31 Jul 2013

HAL is a multi-disciplinary open access archive for the deposit and dissemination of scientific research documents, whether they are published or not. The documents may come from teaching and research institutions in France or abroad, or from public or private research centers.
L'archive ouverte pluridisciplinaire HAL, est destinée au dépôt et à la diffusion de documents scientifiques de niveau recherche, publiés ou non, émanant des établissements d'enseignement et de recherche français ou étrangers, des laboratoires publics ou privés. 
Elsevier Editorial System(tm) for Medical Engineering \& Physics Manuscript Draft

Manuscript Number:

Title: Time-frequency analysis to detect bone fracture in impact biomechanics. Application to the thorax.

Article Type: Paper

Section/Category: Regular Issue Paper

Keywords: thorax, impact, wavelet analysis, fracture detection, piezoelectric transducer

Corresponding Author: $\mathrm{M}$ francois gabrielli,

Corresponding Author's Institution: INRETS

First Author: francois gabrielli

Order of Authors: francois gabrielli; francois gabrielli; Damien Subit, PhD; Erick Ogam, PhD;

Philippe Guillemain, PhD; Richard Kent, PhD; Catherine Masson, PhD

Abstract: Experimental testing is a major source of data to quantify the tolerance of the human body to impact and to develop protection strategies. Correlating the time of rib fractures with the kinematics of the occupant and the action of safety systems would provide valuable data for assessing safety systems and developing injury risk functions. However, methods for determining rib fractures timing are not yet fully developed. Time history analysis of data from multiple strain gauges mounted directly to ribs is commonly used for this purpose, but this method is not very sensitive and the time and cost required to instrument the ribcage with more than 100 strain gauges is prohibitive for many applications. In this study a new approach based on time-scale analysis of signals obtained from piezoelectric transducers (PZT) is reported. A post-mortem human subject was instrumented with four PZT on ribs 3 and 7 bilaterally and exposed to lateral blunt impacts to the shoulder and the chest. The fractures were documented after each test, and a 
Time-frequency analysis to detect bone fracture in impact biomechanics.

Application to the thorax.

\author{
Gabrielli $F^{*} .{ }^{a}$, Subit ${ }^{c}{ }^{c}$, Ogam E. ${ }^{b}$, Guillemain P. ${ }^{b}$, Kent R. W. ${ }^{c}$, Masson C ${ }^{a}$. \\ a Laboratoire de Biomecanique Appliquee, UMRT 24 INRETS, 13916 Marseille \\ Cedex 20, France \\ b Laboratoire de Mecanique et d'Acoustique, UPR CNRS 7051, 13402 Marseille \\ Cedex 20, France \\ c University of Virginia, Center for Applied Biomechanics, 1011 Linden avenue, \\ Charlottesville, VA 22902, USA
}

Word count : 4302 (from introduction to perspectives)

Keywords : thorax, impact, wavelet analysis, fracture detection, piezoelectric transducer

*Corresponding author. Tel.: +33 4 91658000; fax: +33 491658019.

E-mail address: francois.gabrielli@inrets.fr (F. Gabrielli) 


\begin{abstract}
Experimental testing is a major source of data to quantify the tolerance of the human body to impact and to develop protection strategies. Correlating the time of rib fractures with the kinematics of the occupant and the action of safety systems would provide valuable data for assessing safety systems and developing injury risk functions. However, methods for determining rib fractures timing are not yet fully developed. Time history analysis of data from multiple strain gauges mounted directly to ribs is commonly used for this purpose, but this method is not very sensitive and the time and cost required to instrument the ribcage with more than 100 strain gauges is prohibitive for many applications. In this study a new approach based on time-scale analysis of signals obtained from piezoelectric transducers (PZT) is reported. A post-mortem human subject was instrumented with four PZT on ribs 3 and 7 bilaterally and exposed to lateral blunt impacts to the shoulder and the chest. The fractures were documented after each test, and a criterion was developed to process the PZT signals. The criterion consists in detecting in the PZT signal the onset of a high frequency transient generated by the fracture of a rib using the continuous wavelet transform. Two thresholds were successfully determined to detect fractures that occurred (1) on an instrumented rib, and (2) on the adjacent rib. Further development of this method should allow the detection of all rib fractures using only a few PZT.
\end{abstract}




\section{Introduction}

A comprehensive understanding of injury mechanisms remains an important and elusive challenge for designers of vehicle safety countermeasures. Laboratory impact experiments that reproduce the relevant mechanics of field crashes are an essential aspect of this understanding. Correlating the time of bone injury with other events such as the kinematics of the occupant and vehicle, the action of safety systems, and measured deformations or applied loads would provide valuable, unbiased data for assessing safety systems and developing statistically based injury risk functions. The determination of rib fracture timing is of particular interest as these injuries are a good indicator of restraint performance, are frequent, and are potentially dangerous since rib fractures compromise the ability of the chest to protect internal organs critical to life such as the lungs and the heart. Moreover, the capability to monitor rib fractures in the case of an impact to the chest may allow for repeated non-injurious impact tests on a single subject and thus allow for parameterized studies that minimize scatter due to biological variability. Performing a CT-scan after each test to assess the rib fractures, in addition to being extremely expensive, does not provide the required level of sensitivity or specificity. Indeed, the only reliable way to document rib fractures is to perform an in-depth necropsy (Kent et al., 2002), because non-displaced fractures cannot be seen on CT images. Non-displaced fractures may, however, be critical to document because the number and distribution dictates the degree to which the fractures decrease the strength and stiffness of the chest (Kuppa \& Eppinger, 1998; Kent et al., 2004; Yoganandan et al., 2004; Shaw et al., 2007) and changes the injury severity coding (Gennarelli \& Wodzin, 2005).

Sensors to identify the timing and location of both displaced and non-displaced bone fractures in the chest during an impact have not been convincingly demonstrated. Strain gauges are commonly used to achieve this goal (Lebarbe et al., 2005, Kemper et al., 2008; Trosseille et al., 2008) since, at the time of fracture, strain is released and a sudden drop can be observed in the strain measurement. This drop in the strain time-history is used to determine if and when there was a rib fracture (Lebarbe et al., 2005). There are several limitation in this approach. One is the method's dependence on the amplitude of strain in the temporal domain: any attenuation in the signal hampers the ability to detect fracture. This limitation is due to the short detection range of the strain gauges. $A$ fracture has to occur within about $50 \mathrm{~mm}$ of a strain gauge to be reliably detected by this strain gauge (Lebarbe et al., 2005). This critical separation is consistent with the findings of Shaw and Lessley (2007), who also noted that fractures that occurred under the loading device or in the costal cartilage were likewise not reliably detected using strain gages. This spatial attenuation of the signal dictates the use of as many as 5 to 6 gauges per rib to properly map the fracture timing of the entire rib cage. Installing more than 100 strain gauges per chest is an invasive procedure and extremely costly in terms of time, instrumentation, and data acquisition channels. 
An alternative methodology based on time-scale analysis is investigated in the present paper to overcome these limitations. Time-scale analysis (TSA) is a signal processing tool based on wavelet decomposition already used in mechanics to study transient and fast changing signals (Gaul \& Hurlebaus, 1998, Meo et al., 2005, Guillemain \& Kronland-Martinet 1996, Cheng et al., 2003, Knox 2004). ). TSA has been used in biomedical studies (Akay, 1998), for gait analysis (Verdini et al., 2000), and used to localize and indentify impact location on a plate (Gaul \& Hurlebaus, 1989; Meo et al., 2005), but it has never to the authors' knowledge been used for fracture detection in impact biomechanics. The general approach reported here is to use piezoelectric transducers (PZT) to measure rib deformation. PZT transform the mechanical deformation in any direction into a voltage. They are different from strain gauges that measure deformation in only one direction. The goal of this study is to present how TSA can be used to detect rib fractures during an impact test performed on Post-Mortem Human Subjects (PMHS) instrumented with PZT. Six full-scale lateral impact tests were performed on one PMHS (Subit et al, 2009). Ribs 3 and 7 on each side were instrumented with a PZT. The rib fractures were assessed via necropsy after each test. The fracture data were supplemented with the PZT data process. Two fracture thresholds derived from TSA were evaluated to correlate rib fracture occurrence with PZT signals.

\section{Materials and method}

\section{SHPM preparation, experimental data and lesion}

One PMHS (male, $47 \mathrm{~kg}, 173 \mathrm{~cm}$, cause of death: lung cancer) was selected for this study, based on the absence of pre-existing fractures, lesions or other bone pathology, as confirmed by pre-test computed tomography (CT) analysis. The cadaver was obtained and treated in accordance with the ethical guidelines established by the US National Highway Traffic Safety Administration (NHTSA), and all testing and handling procedures were reviewed and approved by an independent oversight committee at the University of Virginia. The subject was screened negative for infectious diseases and stored in a freezer $\left(-15^{\circ} \mathrm{C}\right)$ until it was removed and thawed at room temperature 48 to 72 hours prior to the pretest preparation. Incisions were made at the level of the anterior left and right ribs 3 and 7 (Figure 1). The soft tissues and periosteum on the section of the rib where the PZT (see following section) were to be installed were removed to clear the rib bone. The rib bone was then dried with an isopropanol solution. Next, the cleared rib bone was sprayed with a cyanoacrylate activator (Loctite, \#7455), and the surface of the PZT to be affixed to the bone was covered with cyanoacrylate glue (Loctite, \#382). Finally the PZT was pressed against the bone until the glue hardened. 


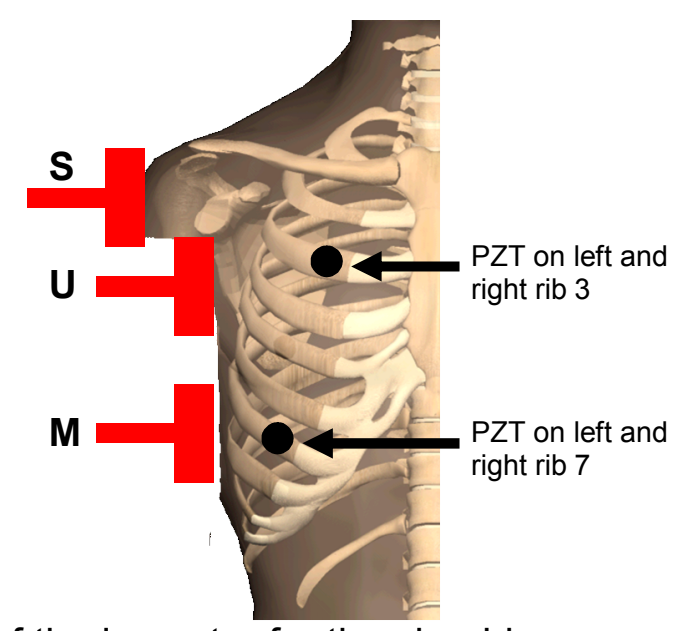

Figure 1: Position of the impactor for the shoulder, upper chest and mid-chest impacts. The PZT were installed on ribs 3 and 7.

The subject was seated on a simplified seat designed to mimic a standard car seat. The impacts were applied using a pneumatic impactor. A catching system was used to prevent interactions with the ground and avoid any artifactual fractures. The impact velocity was $3 \mathrm{~m} / \mathrm{s}$. The subject was successively impacted at 3 levels (Figure 1): shoulder (S) with the arm along the chest, upper chest (U) and mid chest (M), both with the arm at $90^{\circ}$ flexion. Table 1 shows the sequence of impacts on the two sides of the subject. After each test, attempts to detect the rib fractures were made via palpation by a physician-researcher with extensive experience in thoracic biomechanics. A careful necropsy was performed after the tests series to document the rib fractures produced by all the 6 tests.

Table 1: Test matrix and impacted ribs for each impact level (Right and left are defined with respect to the subject). In the $3^{\text {rd }}$ column, RR: right rib, LR: left rib.

\begin{tabular}{|c|c|c|}
\hline $\begin{array}{c}\text { Impact } \\
\text { number }\end{array}$ & Impact location & Ribs impacted \\
\hline 1 & Right Shoulder (RS) & RR1 \\
\hline 2 & Right Upper chest (RU) & RR3 to RR4 \\
\hline 3 & Right Mid chest (RM) & RR5 to RR8 \\
\hline 4 & Left Shoulder (LS) & LR1 \\
\hline 5 & Left Upper chest (LU) & LR3 to LR4 \\
\hline 6 & Left Mid chest (LM) & LR5 to LR8 \\
\hline
\end{tabular}

Each rib fracture was described during necropsy as displaced, non-displaced, mono-cortical or bi-cortical (Table 2, Figure 2). Fractures were found for ribs 3 and below. No fracture was detected after the impacts on shoulder. As expected, not all the fractures were detected by palpation. Seven out of the ten fractures were not displaced. The success rate to detect non-displaced rib fractures is unusually high because the subject was skinny and that facilitated the palpation. 


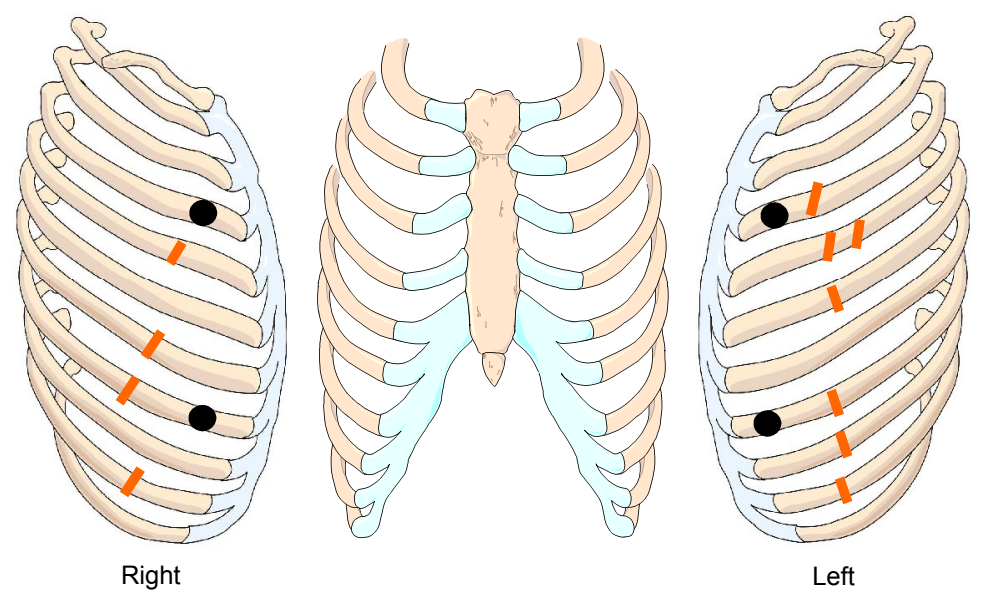

Figure 2: Injuries documented during the necropsy.

Table 2: Repartition of the injuries documented during the necropsy and by palpation after each test.

\begin{tabular}{|c|c|c|}
\hline Impact test & $\begin{array}{c}\text { Fracture detected by palpation \& } \\
\text { autopsy }\end{array}$ & $\begin{array}{c}\text { Fracture missed during } \\
\text { palpation and detected during } \\
\text { autopsy }\end{array}$ \\
\hline RS & None & $\begin{array}{c}\text { Right rib 6 (non displaced) } \\
\text { Right rib 9 (non displaced) } \\
\text { Left rib 5 (non displaced) }\end{array}$ \\
\hline RU & Right rib 4 (non displaced) & \\
\hline RM & Right rib 7 (non displaced) & \\
\hline LS & None & \\
\cline { 1 - 2 } LU & $\begin{array}{c}\text { Left rib 3 (Bi cortical) } \\
\text { Left rib 4 (Mono Cortical, } \\
\text { fragments) }\end{array}$ & \\
\hline LM & $\begin{array}{c}\text { Left rib 7 (Bi cortical, displaced) } \\
\text { Left rib 8 (Non displaced) } \\
\text { Left rib 9 (Displaced) }\end{array}$ & \\
& \multicolumn{2}{|c|}{}
\end{tabular}

\section{Piezoelectric Transducer (PZT)}

PZT have the ability to generate an electric potential in response to applied mechanical stress. A useful PZT electrical model is a voltage source proportional to the applied strain in series with a capacitance $C$. Any resistive load $R$ forms a divider network with a $R C$ high-pass filter characteristic that determines the PZT resonance frequency. For frequencies below the resonance frequency, the PZT gives an output signal proportional to the rate of change of the strain (differentiator system). It is therefore an appropriate sensor to measure transient signals. The PZT for this study was chosen so that its resonant frequency was higher than the upper bound of the data acquisition system (DAS) frequency bandwidth. Figure 3 shows that the PZT was used in the optimum frequency 
range. The signals were sampling at $10 \mathrm{kHz}$ with an Impax DSPT TRAQ-P 12 bitresolution data acquisition system (DSP Technologies, Fremont, CA, USA). A low-pass anti-aliasing filter with a cut-off frequency $F_{c o}$ of $3.3 \mathrm{kHz}$ was applied at the front end of the DAS. The $9.9 \mathrm{~mm}$-diameter PZT with a resonant frequency of $7 \mathrm{kHz}$ (model AB1070B, Projects Unlimited, Dayton, OH, USA) was found to be appropriate for this study because of its size (it fits on a rib bone) and its frequency response (Figure 3).

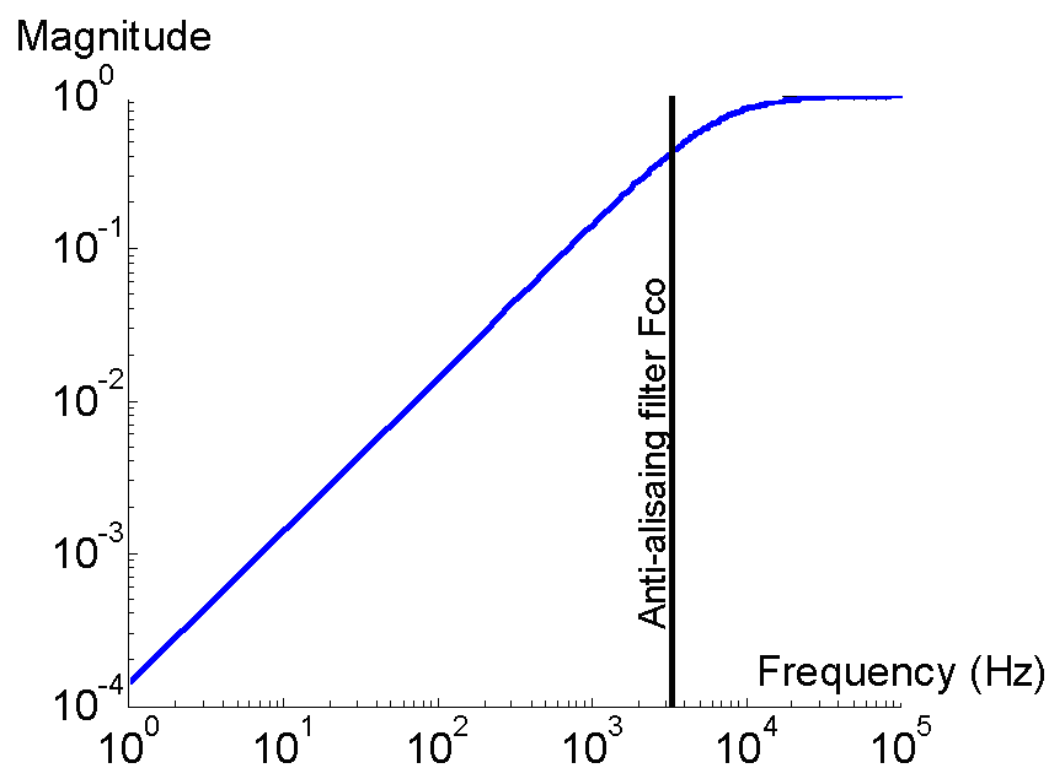

Figure 3: Frequency response of the PZT

Time scale analysis

In order to quantify the transient within the PZT signal we propose a method based on the Continuous Wavelet Transform (CWT). The continuous wavelet transform of a signal $x(t)$ is defined by:

$C W T_{x}^{\Psi}(b, a)=\int_{-\infty}^{+\infty} x(t) \frac{1}{\sqrt{a}} \bar{\Psi}\left(\frac{t-b}{a}\right) d t$ where $\bar{\Psi}$ indicates the complex conjugate of $\Psi$, and $\Psi$ is the analyzing wavelet.

The wavelet transform is fundamentally a correlation between the signal $x(t)$ and a wavelet function $\Psi(t)$ translated by $b$ and dilated by $a$. The chosen mother wavelet function $\Psi(t)$, known as the "Morlet" wavelet, is a modulated Gaussian:

$$
\Psi(t)=e^{-\frac{t^{2}}{2}+j 2 \pi f_{0} t}
$$


The Morlet wavelet is localized around time $t=0$ and its Fourier transform is centered on frequency $f_{0}$. It is easily understood that the function $\Psi((t-b) / a)$ is localized around $t=b$ and its Fourier transform around $f=f_{0} / a$. Hence $|C W T(b, a)|$, the magnitude of $C W T$, represents the square root of the power density of the signal $x(t)$ around the time $t=b$ and the frequency $f=f_{0} / a$.

The $C W T$ was chosen for its ability to operate at every scale up to the defined maximum. In addition, as the wavelet analysis is smoothly done along the signal, it allows for the localization of any change in the frequency content or any transient.

The $C W T$ was applied to each PZT signals. Figure 4 shows a typical time-history response of a PZT. The output of the $C W T$ analysis is called a scalogram, which is a three-dimensional representation where time is on the $x$-axis, frequency (related to scale) is on the $y$-axis, and magnitude is on the z-axis (Figure 5).

The evaluation of the strength of the transient is based on the fact that the shorter the transient in time, the richer its frequency content (Figure 5). The limit is the Dirac pulse, which has a zero length in time and an infinite frequency width.

When a fracture occurs, strains in the material are relieved, which generates a high frequency wave in the bone, leading to an increase in the signal frequency bandwidth. Therefore the occurrence of a fracture can be detected by tracking the frequency bandwidth of the PZT signals as a function of time. A criterion was developed to determine the time of fracture by correlating the changes in the frequency width with the fractures documented after each test. 


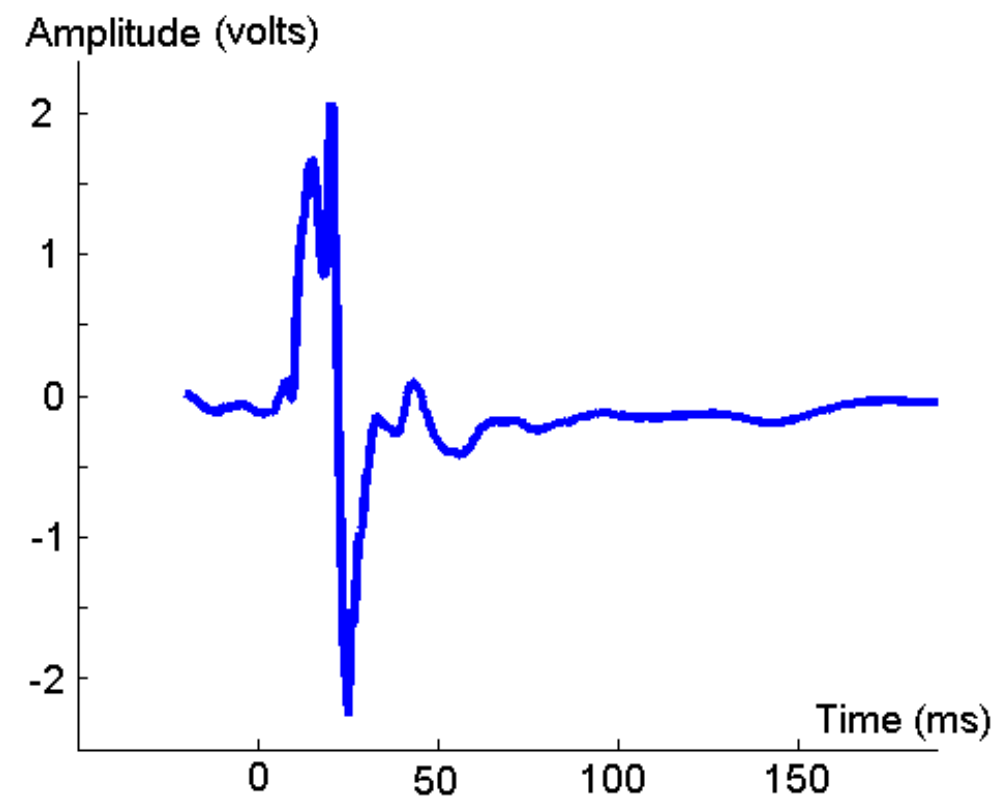

Figure 4: Example of time-history PZT response to chest impact

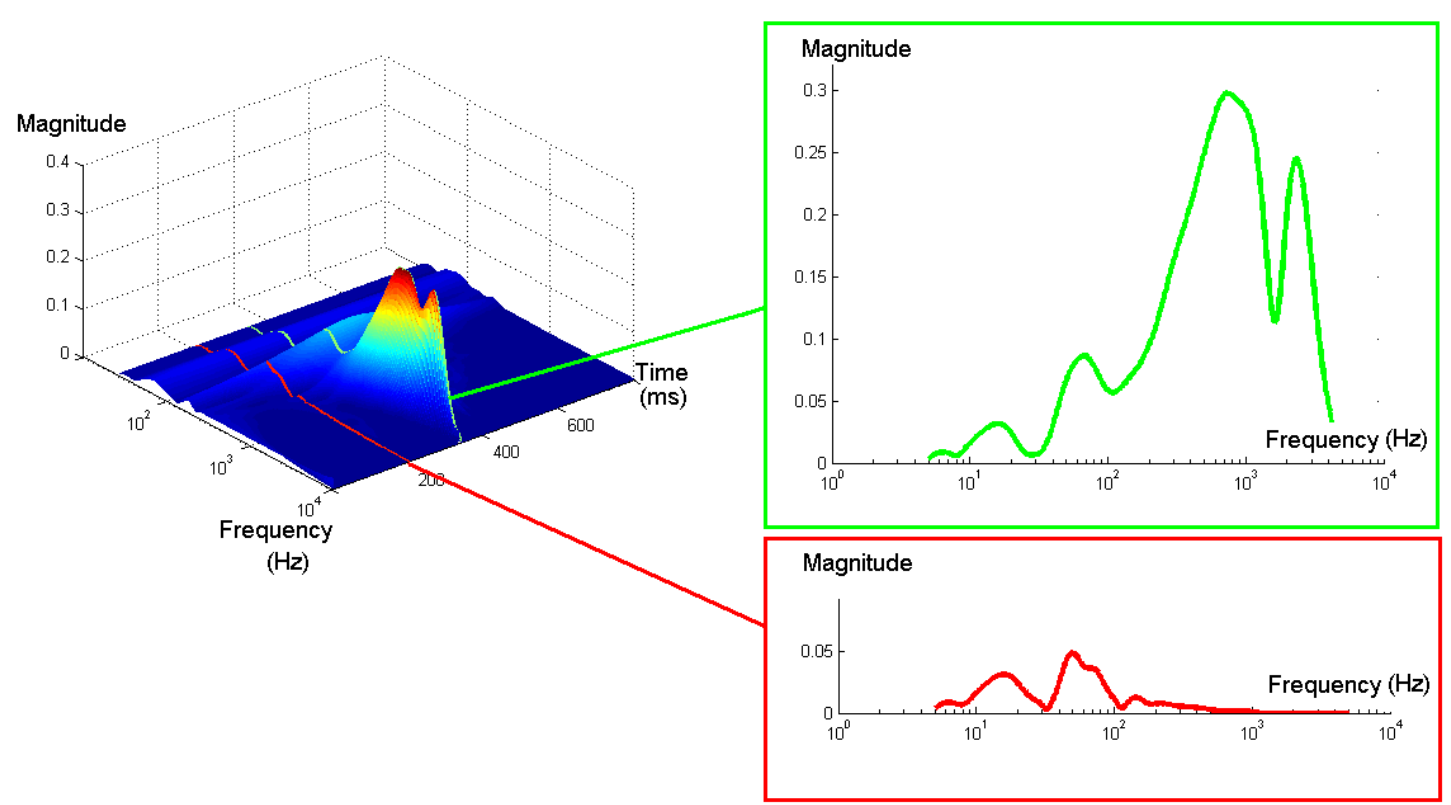

Figure 5: Scalogram selection at two separate instants with different upper frequency spectrum

The methodology consists of seeking a wide band of energy at a given time. It is adapted from a methodology proposed by Antoine et al. (1997) and Kang \& Birtwhistle (2003). The strength of a transient is defined as the integral of the 
module of the $C W T$ from the finest scale (corresponding to the maximum frequency) up to a cut off scale $a_{c}$ (corresponding to a cut off frequency $f_{c}$ ):

$$
\delta(b)=\int_{a_{\min }}^{a_{c}}\left|C W T_{x}(a, b)\right| d a
$$

The resulting criterion reflects both the instantaneous bandwidth and the signal power. The greater the magnitude of the criterion, the greater the energy in the upper section of the spectrum and the more transient the signal. The more transient the signal, the more likely it is to reflect the occurrence of a fracture. Indeed transient signal depends on the structure: when a rib breaks, there is a sudden change in the length of the structure to which the PZT is affixed. After fracture, the PZT remains on a smaller structure, and the energy released by the fracture generates a highly oscillatory signal. The phenomenon was analyzed by plotting the function $\delta$ for all the PZT for each impact test as a function of the translation integer $b$, which is the translation of the wavelet and consequently is equivalent to time.

Thresholds were defined for the criterion $\delta$ by correlating its value with the rib fracture occurrences (Table 2). Any time the criterion exceeded the threshold level $T$, we required that a fracture had to occur in the vicinity of the transducer. The values of the threshold $T$ and the parameter $a_{c}$, which delimits the low and high frequencies, were determined so that the value of $\delta$ was greater than $T$ when a fracture occurred.

\section{Results}

\section{PZT signals}

It was noted that the PZT on Left Rib 3 malfunctioned during all tests. The LR3 signal was therefore removed from the analysis.

Examples of PZT signals are shown Figure 6. These signals were recorded by the PZT on Right Rib 3 during the tests 1, 2 and 3. Fracture of the right rib 4 was documented after the right upper chest impact (Fig. 6(b)) and fracture of the right rib 7 was documented after right mid-chest impact (Fig. 6(c)). Analysis of the time-history signals presented in Figure 6 does not provide sufficient information to discriminate whether a fracture occurred during the impact. 


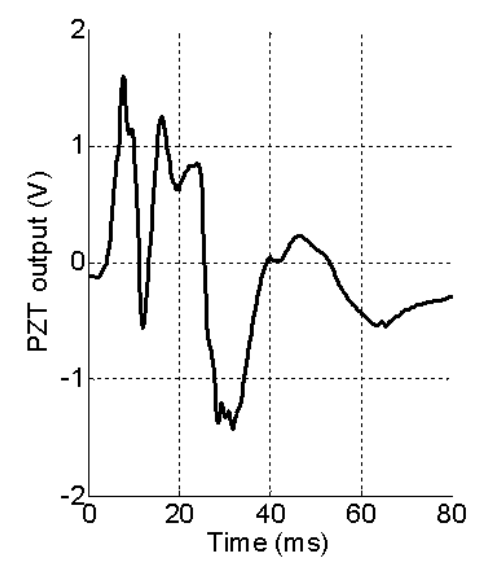

(a)

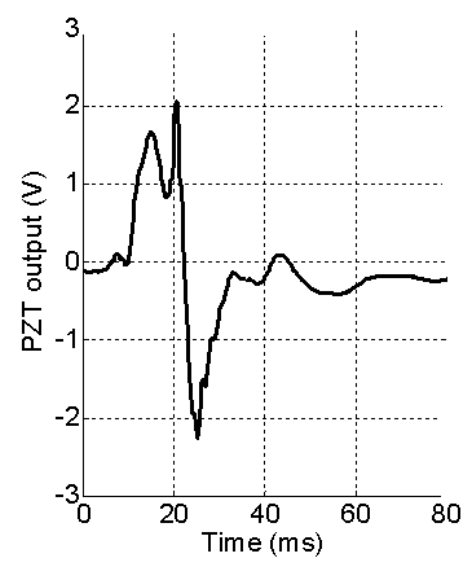

(b)

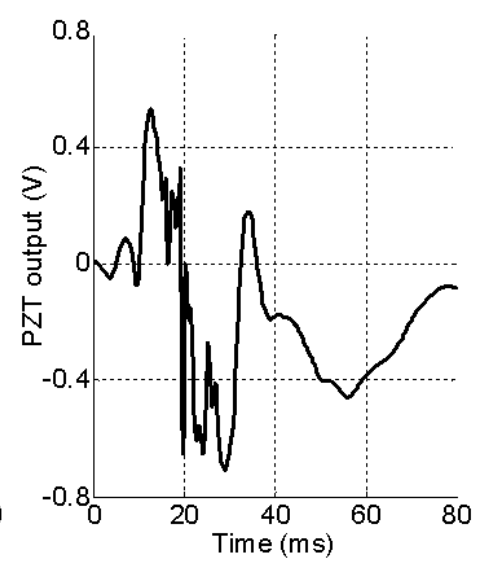

(c)

Figure 6: Time-history of PZT signals cannot discriminate the occurrence of a fracture (Right rib 3 PZT signals for tests (a) shoulder, (b) upper chest and (c) mid-chest).

\section{Transient criterion}

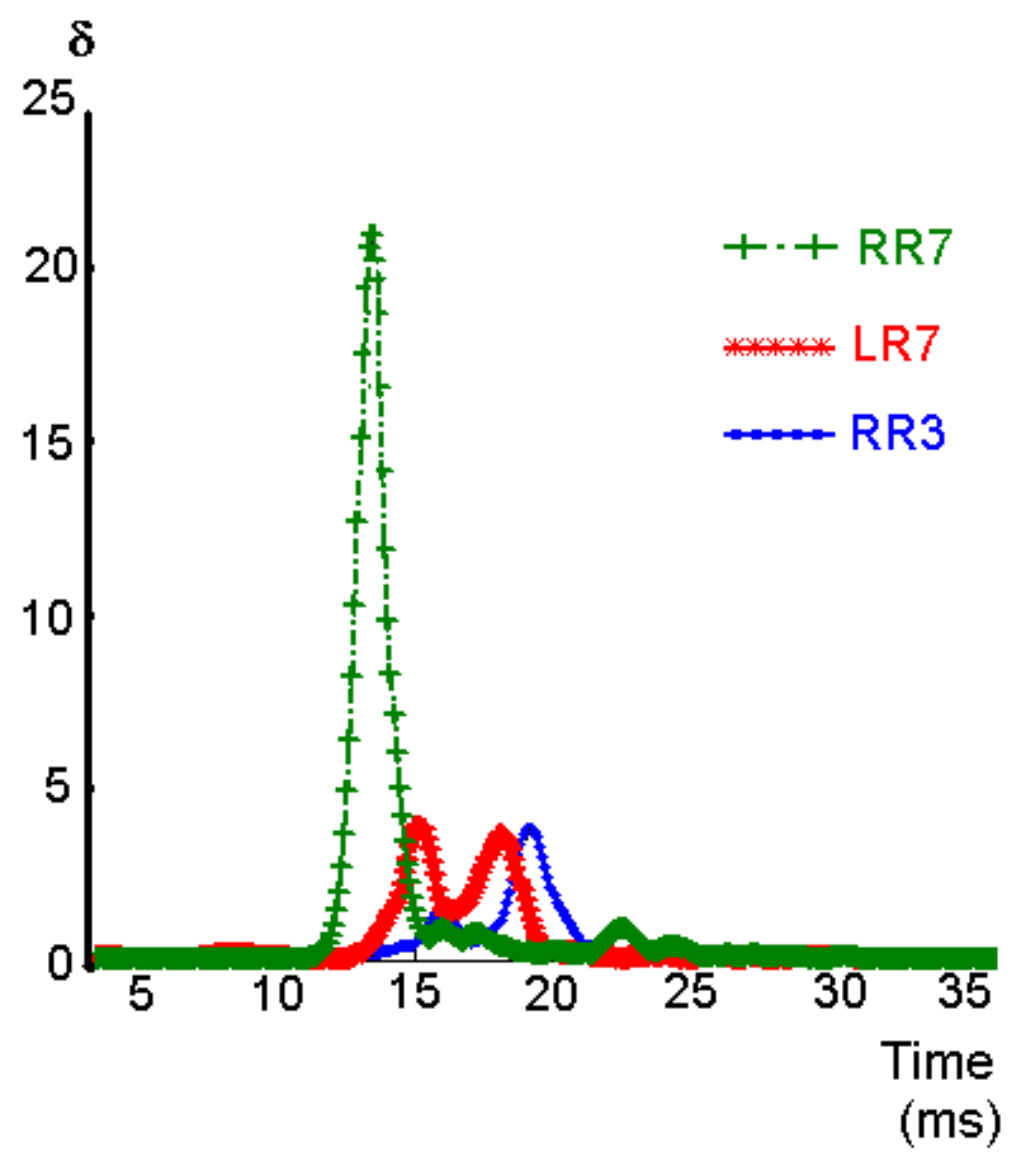


Figure 7: Values of the criterion for the impact to the right midchest calculated for the PZT signals on RR7 where a fracture was documented, RR3 and LR7 for $f_{c}=1145 \mathrm{~Hz}$ ).

Figure 7 shows the resulting criterion for the right mid-chest impact (test \#3). For this test, a fracture was detected by the PZT on RR7. The criterion exhibits a large peak for RR7 and two smaller and delayed peaks for the PZT further away from the broken rib (LR7 and RR3). It shows that two thresholds for the criterion value can be determined to discriminate fractures that occurred on the rib to which the PZT is attached or on another rib. When the criterion reaches the first threshold level, referred to as close rib level or CRL, the corresponding sensor detects a fracture that occurred on a close rib. When the criterion reaches the second level, referred to as the sensor rib level or SRL, the rib to which the sensor is mounted breaks.

The parameter $a_{c}$ sets the bandwidth for the computing of the transient criterion and therefore its ability to discriminate mere impact without fracture from impact inducing fractures on the sensor rib or on a distant rib. One must be certain that a mere impact would generate a little transient signal that would have a criterion low enough not to be confused with an impact inducing fracture. Moreover the criterion level of an impact inducing a fracture on a distant rib must be significantly lower than the criterion level of an impact inducing a fracture on the sensor rib. A parametric study was realized in order to determine the optimal $a_{c}$ that would discriminate occurrences of fracture on the sensor rib from fracture on a distant rib. The minimum criterion values for the PZT attached to a rib that broke, and the maximum values for the PZT attached to a rib close to a rib that broke where calculated for $a_{c}$ corresponding to frequencies between $339 \mathrm{~Hz}$ and 2578 HZ (Figure 8). 


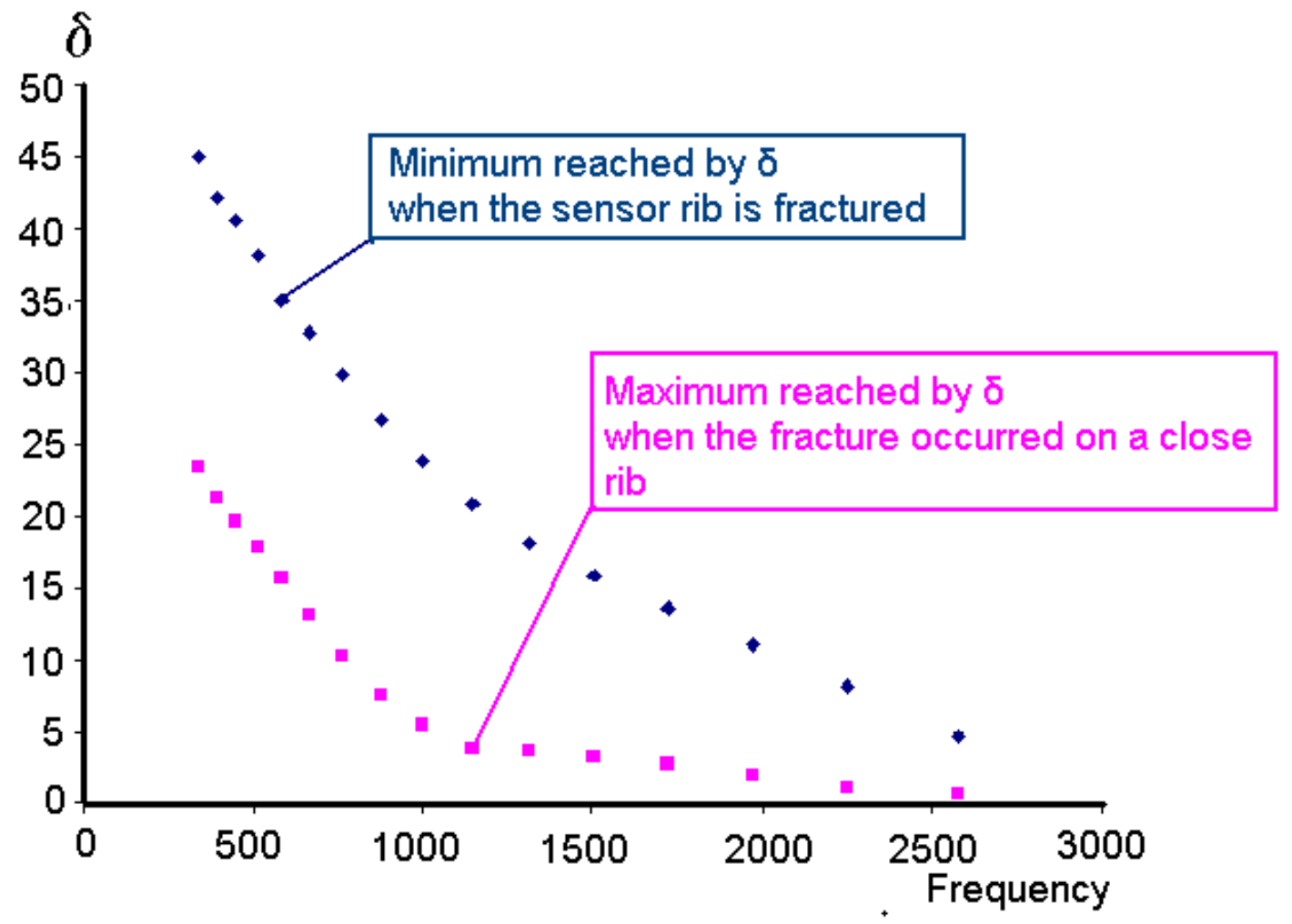

Figure 8: Variation of the criterion levels as a function of the cut off frequency $f_{c}$

The criterion $\delta$ is the integration from the lowest scale up to the cut off scale, $a_{c}$. Therefore, as expected, the criterion value decreased with the frequency since the integration domain widens as cut-off scale increases. For the range of tested cut-off frequencies, the criterion is able to discriminate a fracture occurring on a close rib from that occurring on the sensor rib. The optimum cut off frequency maximizes the difference between the criterion level of a fracture on a close rib and the criterion level of a fracture on the sensor rib and is therefore set at $1145 \mathrm{~Hz}$

Sensor Rib Level and Close Rib Level were further investigated for a scale value $a_{c}$ corresponding to a frequency of $1145 \mathrm{~Hz}$ (Table 3). The Right Rib 3 PZT detected a fracture on a close rib for all the impacts except for the shoulder impacts. The Right rib 7 PZT detected a fracture on its very rib during the right mid chest impact. Finally, the Left Rib 7 PZT detected a fracture on a close rib during the left upper chest impact and a fracture on its very rib on the left mid chest impact.

Table 3: Successful fracture detection ( $a_{c}$ corresponding to $1145 \mathrm{~Hz}$ ) and $\mathrm{SRL}=$ 10 and $\mathrm{CRL}=1$ 


\begin{tabular}{|c|c|c|c|c|c|c|}
\hline & RS & RU & RM & LS & LU & LM \\
\hline Test \# & 1 & 2 & 3 & 4 & 5 & 6 \\
\hline RR3 & - & Close rib & Close rib & - & Close rib & Close rib \\
\hline RR7 & - & - & Sensor rib & - & - & - \\
\hline LR7 & - & - & Close rib & - & Close rib & Sensor rib \\
\hline $\begin{array}{c}\text { Palpated } \\
\text { Fractures }\end{array}$ & Right rib 4 & Right rib 7 & Left rib 3, 4 & Left rib 7,8 9 \\
\hline $\begin{array}{c}\text { Fractures } \\
\text { not } \\
\text { detected } \\
\text { during } \\
\text { palpation }\end{array}$ & \multicolumn{7}{c}{$\begin{array}{c}\text { Right rib 6 (non displaced) } \\
\text { Right rib 9 (non displaced) } \\
\text { Left rib 5 (non displaced) }\end{array}$} \\
\hline
\end{tabular}

\section{Instant of occurrence}

If a PZT is placed near the fracture location, the instant of fracture can be determined from the raw PZT time-history signal (Right mid-chest impact, Figure 9(a)), without requiring any processing or consideration of the frequency content. This fortuitous result in one of the PMHS impacts allows for an assessment of any time delay in fracture detection that may be introduced by the TSA. Both the raw PZT signals and the corresponding value of the $\delta$ criterion show that the right rib 7 fracture occurred at time $13 \mathrm{~ms}$ (Figure $9 \mathrm{a}$ and b). The RR3 and LR7 PZT detected this fracture as well. The time differences in the peaks provide an estimate of the time of flight of the transient: LR7 detected the fracture after $2 \mathrm{~ms}$, and RR3 after $6 \mathrm{~ms}$. The further the PZT from the fracture location, the greater the time delay: a time window of fracture occurrence can then be set from the time of flight and the last peak of the criterion.

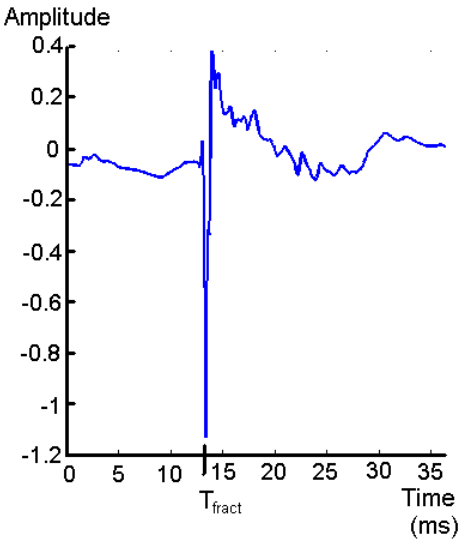

(a)

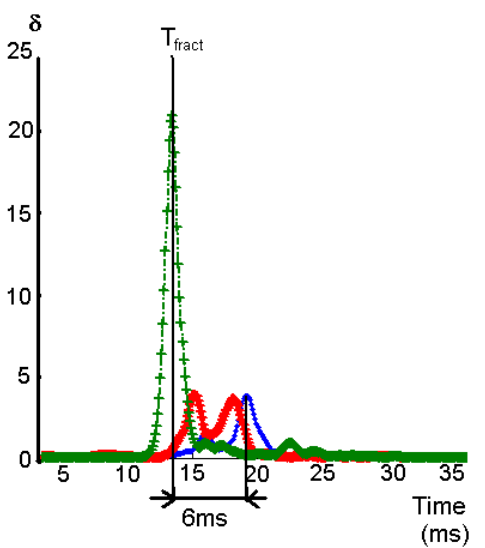

(b)

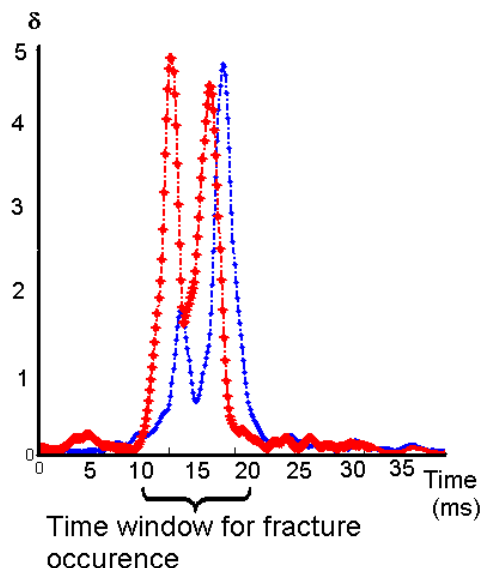

(c) 
Figure 9: Right mid chest impact (a) PZT signal of RR7 with time of fracture at 13ms. (b) Criterion for RR7 ( -+ green), LR7 ( -* red) and RR3 ( -. blue) with a delay of $+6 \mathrm{~ms}$. (c) Criterion for RR3 ( -. blue) and LR7 ( ${ }^{*}$ red) illustrating the time window of fracture occurrence

\section{Discussion}

\section{Fracture detection}

Several successive impact tests were performed on a thorax with PZT mounted to selected ribs. The injury report from the necropsy shows that a physician can easily miss non-displaced fractures during palpation and that it is therefore critical to have a tool that has a high success in rib fracture detection. The results of the present study showed that a methodology using PZT and time scale representation is promising for determining the time window of the fracture occurrence.

A major improvement compare to the strain gauge-based method is the ability to detect a fracture even if it occurred on an uninstrumented rib. Indeed, earlier work has shown that the detecting strain gauge needs to be on the broken rib and moreover within approximately $50 \mathrm{~mm}$ of the fracture location. The preliminary work presented here indicates that it may be possible to use approximately $20 \mathrm{PZT}$ compared to more than 100 strain gauges to attain similar spatial resolution for fracture detection (Kemper et al., 2008; Trosseille et al., 2008). Ongoing work is being conducted to determine how to reduce the number of PZT while detecting all the fractures.

\section{PZT location}

For the subject used in this study, the fractures occurred on the anterior section of the rib, posterior to the PZT location. After fracture, the PZT remained attached to the short rib segment connected to the sternum. The fracture progression shown in Table 3 indicates that once an instrumented rib is broken the PZT attached to it loses the ability to detect a fracture on an adjacent rib. Two explanations are proposed to explain this behavior. First, once the fracture has occurred, the stiffness of the rib is greatly reduced. Therefore its ability to transmit high frequency waves decreases. Second, the transient signal generated by a fracture travels through the posterior rib and the spine, and not through the sternum. Indeed the sternum is surrounded by costal cartilage that behaves as a damping material and could act as a low pass filter. In contrast, the spine may provide a stiffer structure than the sternum for wave propagation since each rib attached to two vertebrae through the superior and inferior costal facets. Consequently, future work should assess the performance of the PZT when 
mounted to the most posterior part of the rib as better fracture resolution may occur.

Sensitivity to the parameter $a_{c}$

Results showed that a scale level $a_{c}$ could be found that separates high frequency from low frequency and that could detect a transient phenomenon in the signal. The scale level for the sensor-mounted rib level was straightforward to determine: the criterion always reached a very high level when the rib to which it was attached fractured. The challenge was more on the fine tuning of $a_{c}$ and $\mathrm{CRL}$. Transient propagation within biological environment implies damping of soft tissues and joint connection between bony structures. High frequency composition of a transient is likely to be attenuated as the transient propagates through many biological structures. Thus, for $a_{c}$ too low, the band of frequencies considered as high would be very narrow: the PZT would be too restrictive and would lose the capability of seeing a fracture occurring on a close rib.

\section{Determination of fracture time}

In the current analysis, the time of fracture was defined as the time when the criterion reached a maximum. As a maximum in the criterion reflects an instant of high speed variation in the signal, the assumption that it is the instant of fracture seems reasonable. A more conservative assumption is that the fracture occurs within the time the criterion is above the threshold (Figure 10). If a time window is considered instead of a time instant, then several parameters have to be considered that widen the fracture window. One is the time of flight of the transient information from the fracture to the $\mathrm{PZT}$, especially if the sensor is not on the fractured rib. A second parameter is the width of the Gaussian windows of the $C W T$, which increases with the scaling parameter $a$. Thus a higher $a_{c}$ would widen the peak of the criterion.
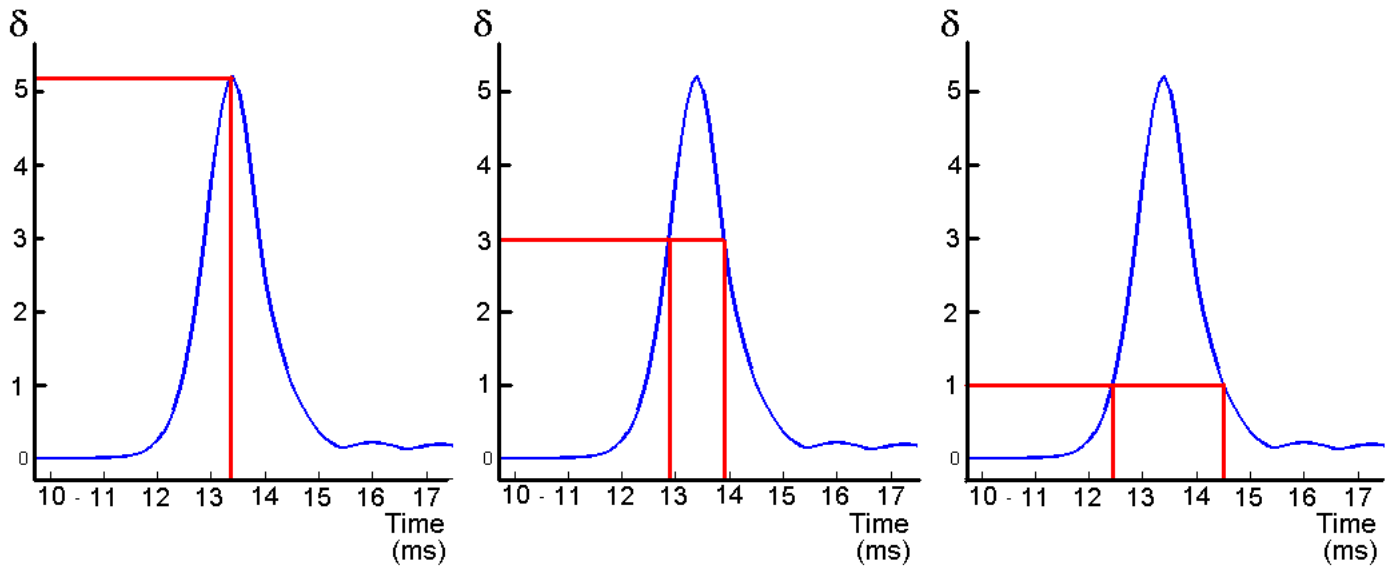

Figure 10: Window width depends of threshold. 
Finally, the physics of the event - onset of a crack, propagation and maybe complete fracture - has not been taken into account yet. The frequency response of the fracture process certainly varies as the crack propagates in the bone. This needs to be studied to describe the characteristics of the transient itself and how the value of the criterion relates to the fracture process.

\section{Limitations}

The main purpose of the paper was to investigate the feasibility of the detection of a fracture using a reduced number of sensors compared to the strain-gauge technique used in the past. Signal analysis of PZT mounted on the ribs of a laterally struck PMHS indicated that fractures of both instrumented and uninstrumented ribs can be detected. One limitation to the methodology is that it is not yet possible to identify precisely the fractured rib if not instrumented. In other words, it it possible to know that a fracture occurred on a nearby rib, but not which nearby rib. The capability of the methodology to localize fracture on adjacent ribs could lead to an anatomic tracking of the location of the fracture, but additional work is needed to develop that aspect of the method. Figure 9(b) shows a delay between a PZT detecting a fracture on the rib to which it is mounted and the PZT detecting the fracture from a nearby rib. The magnitude of the signal's time of flight through the several biological structures of the rib cage could be further investigated as a method for localizing fractures. Such knowledge could improve our knowledge of lesion mechanisms of the rib cage and could lead to an optimum distribution of the PZT sensors along the rib cage. On going work is being done to complete this theory.

A second limitation to the study is the use of a single PMHS. It is possible that the injury threshold criterion could be subject specific. The energy measured at time of the fracture depends not only on the position of the sensors on the rib (a sensitivity analysis is required to estimate the influence of the PZT location on the measured signal) but also on the mechanical properties of the rib material and surrounding soft tissues, which are subject-dependent. Thus the two thresholds that were determined from our methodology may not apply to other PMHS. Further analysis of the fracture process is required to better describe the link between the physics of the fracture process and the signal measured by the PZT.

\section{Conclusion}

This study introduced a novel methodology to determine rib fracture time in impact biomechanics testing. Contrary to the common method that relies on time- 
history analysis of strain-gauge data, the proposed method uses an in-depth analysis of a PZT sensor signal's frequency content based on time-scale analysis. This new method shows promise for requiring fewer sensors than the strain gauge-based method as the sensors have the capability to detect fracture even on uninstrumented ribs. The fracture threshold of the parameter $a_{c}$, which delimits low and high frequencies, needs to be tuned to detect fracture on instrumented and uninstrumented ribs. Likewise, the underlying fracture mechanics that generate the wave measured by the PZT need to be better understood to determine if the thresholds SRL and CRL are subject-specific. The proposed method demonstrates the capability of time scale signal processing to characterize a transient signal within a time signal and thus a way to detect rib fractures with a small number of sensors.

\section{Conflict of interest statement}

None of the authors have any commercial relationships which represent a past, present or future conflict of interest

\section{Acknowledgement}

The authors wish to thank Hervé Guillemot (University of Virginia, Virginia, USA, and Université de Valenciennes et du Hainaut Cambrésis, Valenciennes, France, for his contribution in the subject instrumentation and injury documentation, Sonia Duprey (Laboratoire de Biomécanique et Mécanique des Chocs, Lyon, France, and Sabrina Lau (University of Virginia, Virginia, USA) for their help with testing.

\section{Bibliography}

Gennarelli, T.A. and Wodzin, E., 2005. The Abbreviated Injury Scale, 2005, Association for the Advancement of Automotive Medicine (AAAM), Barrington, Illinois, USA.

Crandall, J., Kent, R., Patrie, J., Fertile, J., Martin, J., 2000. Rib fracture patterns and radiologic detection-a restraint-based comparison. Annual proceedings / Association for the Advancement of Automotive Medicine, 235-59.

Kemper, A.R., McNally, C, Kennedy, E.A., Manoogian, S.J., Duma, S.M., 2008. The influence of arm position on thoracic response in side impacts. Stapp Car Crash Journal 52, 379-420.

Kent, R. W., Crandall, J., Patrie, J., Fertile, J., 2002. Radiographic detection of rib fractures: a restraint-based study of occupants in car crashes. Traffic Injury Prevention 3, 49-57. 
Kent, R, Lessley, D, Sherwood, C., 2004. Thoracic response to dynamic, nonimpact loading from a hub, distributed belt, diagonal belt, and double diagonal belts. Stapp Car Crash Journal 48:495-519.

Kuppa, S, Eppinger, R., 1998. Development of an improved thoracic injury criterion. Stapp Car Crash Conference 42, paper 983153.

Trosseille, X., Baudrit, P., Leport, T., Vallancien, G., 2008. Rib cage strain pattern as a function of chest loading configuration, Stapp Car Crash Journal 52, 205-231.

Lebarbe M., Potier P., Baudrit P., Petit P. , Trosseille X., Vallancien G., 2005, Thoracic Injury Investigation using PMHS in Frontal Airbag Out-of-Position Situations, Stapp Car Crash Journal, Vol. 49, 323-342

Ph. Guillemain, R. Kronland-Martinet., Characterization of acoustic signals through continuous linear time-frequency representations. Proceedings of the IEEE, special issue on Wavelets, Vol 84 (4) April 96, pp561-585.

Gaul L., Hurlebaus S.,1998. Identification of the impact location on a plate using wavelets, Mechanical Systems and Signal Processing, Volume 12, Issue 6, 783795

Meo M., Zumpano G., Piggott M., Marengo G.,2005. Impact identification on a sandwich plate from wave propagation responses,Composite Structures, Volume 71, Issues 3-4, 302-306

Cheng Z., Pellettiere J., 2003. Correlation analysis of automobile crash responses based on wavelet decompositions, Mechanical systems and signal processing, volume 17 , issue $6,1237-1257$

Knox T., 2004. Validation of earplug accelerometers as a means of measuring head motion, SAE technical paper series, Proceedings of the 2004 SAE Motorsports Engineering Conference and Exhibition

Akay, 1998. Time Frequency and Wavelets in Biomedical Signal Processing, IEEE Press Series on Biomedical Engineering

Antoine J-P., Barachea D., Cesar R.M. Jr., da Fontoura Costa L., 1997. Shape characterization with the wavelet transform, Signal Processing 62, 265-290

Kang P., Birtwhistle D., 2003. Characterisation of vibration signals using continuous wavelet transform for condition assessment of on-load tap-changers, Mechanical Systems and Signal Processing,17(3), 561-577 
Yoganandan, N., Pintar, F., Gennarelli, T., 2004. Small female-specific biomechanical corridors in side impacts. Proc. International Research Council on the Biomechanics of Impact (IRCOBI) pp. 137-152.

Shaw, G., Lessley, D., Evans, J., Crandall, J., Shin, Joeho, Portier, P., Paoloni, G., 2007. Quasi-static and dynamic thoracic loading tests: cadaveric torsos.

Proc. International Research Council on the Biomechanics of Impact (IRCOBI) pp. 325-352.

Shaw, G. and Lessley, D., 2007. Quasi-static and dynamic thoracic loading tests: cadaveric torsos. Technical Report, National Highway Traffic Safety Administration, Department of Transportation, Washington, DC, USA.

Subit D., Duprey S., Lessley D., Lau S., Guillemot H., Kent R., 2009. Response of the torso in side and oblique impacts under constant impact velocity, Traffic Injury and Prevention, in revision. 


\title{
${ }^{*}$ Referee Suggestions
}

\author{
James Funk \\ BRC - Biodynamic Research Corporation \\ 5711 University Heights Blvd., Suite 100 \\ San Antonio, TX 78249, USA \\ jfunk@BRCONLINE.COM
}

\section{Eric Kennedy}

Assistant Professor,

Biomedical Engineering Department

171 Breakiron Engineering

Bucknell University

Lewisburg, PA 17837

eric.kennedy@bucknell.edu

Xavier Trosseille

LAB PSA Peugeot-Citroën RENAULT, 132 rue des Suisses,

92000 Nanterre,

France.

xavier.trosseille@lab-france.com 